\title{
Versorgungsdefizite in der pneumologischen
}

M. Schmitz Rehabilitation

\author{
Care Deficits in Pneumological Rehabilitation
}

Der Sachverständigenrat für die konzertierte Aktion im Gesundheitswesen legte am 30.08.2001 den Band III „Über-, Unter- und Fehlversorgung“ seines Gesamtgutachtens „Bedarfsgerechtigkeit und Wirtschaftlichkeit“ vor, in dem grundlegende Überlegungen und Definitionen zur Bedarfsgerechtigkeit der Versorgung mit Gesundheitsgütern und daraus ableitend zur Über-, Unter- und Fehlversorgung angestellt werden [1]. Er berichtete über Konzepte und Ergebnisse einer Befragung von 300 wissenschaftlichen Organisationen, Körperschaften und Selbsthilfeeinrichtungen des Gesundheitswesens, verglich Krankheits- und Wirkungsunterschiede zwischen Ost- und Westdeutschland nach der Wiedervereinigung und analysierte krankheitsübergreifende Struktur- und Qualitätsprobleme bei der Versorgung chronisch Kranker.

Über unterschiedliche Krankheiten- und Patientengruppen hinweg identifizierte der Rat konvergente Muster überholter Paradigmen und Versorgungsgewohnheiten, die eine effizientere Versorgung chronisch Kranker behinderten:

1. die Dominanz einer auf akute, episodenhafte und eindimensionale Krankheitsformen ausgerichteten kurativen Versorgung mit einem entsprechend strukturellen Versorgungsgrad,

2. die somatische Fixierung des Gesundheitssystems,

3. die mangelnde Präventionsorientierung des deutschen Systems,

4. eine Aktiv-/Passivproblematik, die den chronisch Kranken als passiven Empfänger von medizinischen Leistungen („Reparatur, Kur und Schonung“" sieht,

5. eine unzureichende Information, Schulung und Partizipation,

6. der Mangel an interdisziplinären und flexiblen Versorgungsstrukturen,

7. das Abweichen von Grundsätzen an evidenzbasierter Versorgung,

8. inadäquate Anreizsysteme, und
9. eine unzureichende Berücksichtigung der speziellen Versorgungsbedürfnisse chronisch Kranker in der Qualifikation und Sozialisation der Gesundheitsberufe.

Der Rat hatte bereits in Band I, Kap. III des Gutachtens 2000/2001 eine stärkere Einbeziehung der Nutzer, also der Bürger, Versicherten und Patienten, als dritte Kraft im Gesundheitswesen gefordert. Dementsprechend griff er bei der jetzigen Analyse auf eine umfassende Befragung von 69 Betroffenenorganisationen zurück, von denen 43 antworteten. Da Selbsthilfebewegungen in Deutschland zwischenzeitlich wesentlich zu einer neuen, veränderten Rolle des Patienten im Hinblick auf seine Unterstützung und Interessensvertretung beitrugen, hatte der Rat die Befragung auf Institutionen der Selbsthilfe ausgedehnt. Dabei waren für ihn u.a. folgende Zielvorstellungen leitend:

1. Die Gruppe chronisch Kranker und Behinderter sei im besonderen Maße auf Hilfen aus unterschiedlichen Bereichen der gesundheitlichen Versorgung, wie z.B. Akutmedizin, langfristige medizinische Behandlung, Rehabilitations-, Pflege- und Sozialunterstützung angewiesen.

2. Die Berichte der Betroffenen seien besonders geeignet, auch das Zusammenwirken unterschiedlicher Sektoren des Gesundheitswesens zu beleuchten.

3. Der Rat sehe gerade in der Authentizität der Berichte von Betroffenen einen unverzichtbaren Beitrag für die Bewertung der Qualität des Versorgungssystems.

4. Somit könnten die Aussagen der befragten Gruppen als sensibler Indikator für die Versorgungsqualität gewertet werden.

5. Dabei nahm der Rat angesichts dieser Vorzüge eine ggf. mangelnde Repräsentativität der Aussagen in Kauf.

Die befragten Institutionen sahen Mängel in nahezu allen Versorgungsaufgaben und -bereichen des Gesundheitswesens hinsichtlich der Leistungsqualität, vorgehaltener Strukturen, Orga- 
nisation und Kooperation. Qualitätsmängel wurden vorrangig in der ambulanten Versorgung, sowohl bei Ärzten, Pflegeteams als auch weiteren Gesundheitsberufen gesehen. Deutlich ist die Bemängelung der unzureichenden Dichte, also einer nur mangelhaft dem Bedarf angepassten Vielfalt vorgehaltener Versorgungsformen. Es fehlten Kompetenzzentren, Spezialambulanzen und Praxen für häufige chronische Krankheiten.

Der Bereich der ambulanten Rehabilitation wurde v.a. für ältere Patienten für unzureichend erachtet. Als zentrales zu Unter- und Fehlversorgung führendes Problem wurde mit hoher Übereinstimmung der Betroffenenorganisationen die mangelnde Kooperation und eine unzureichende sektoren- bzw. schnittstellenübergreifende Versorgung festgestellt. Die Bildung nahtloser, zielorientierter Versorgungsketten, bestehend aus Prävention, Kuration, Rehabilitation, sozialer Reintegration, Hilfsmittelanpassung und Schulung, auf die chronisch Kranke und Behinderte im besonderen Maße angewiesen seien, würden dadurch verhindert, erheblich eingeschränkt oder dysfunktional deformiert. Daraus ergäben sich an Zuständigkeits- und Bereichsgrenzen für Patienten schwerwiegende Versorgungsbrüche. Dabei erachtet es der Rat als unzumutbar, dass sektorübergreifende Verbundleistungen häufig dem Patient selbst überlassen blieben, der zudem noch unzureichend informiert sei.

In den fortgeschrittenen Industrienationen stellt die angemessene Versorgung chronisch Kranker die wichtigste und größte Herausforderung für das Gesundheitswesen dar. $50 \%$ der ambulant und stationär Behandelten in Deutschland sind chronisch krank. Chronisch Kranke verursachen als „Dauer- und Vielnutzer“ einen erheblichen Teil der direkten und indirekten Kosten im sozialen Versorgungssystem, v.a. im Gesundheitswesen. Die untersuchten Indikationen machen dabei ${ }^{2} / 3$ aller Krankheitskosten des Systems aus. Aufgrund der demographischen Alterung der Bevölkerung und der damit im Zusammenhang stehenden Krankheitslast, sowie des medizinischen Fortschritts wird die Bedeutung chronischer Erkrankungen in Zukunft weiter zunehmen. Die Bedeutung chronischer Krankheiten ergibt sich darüber hinaus aus ihrer Komplexität und den damit verbundenen Anforderungen an eine angemessene Organisation in der Abstimmung der unterschiedlichen Behandlungsansätze.

Zur Bewältigung dieser Aufgaben stellt der Rat klare Anforderungen an die Versorgung chronisch Kranker:

1. bedarfsgerechte Versorgung entsprechend des multidimensionalen Charakters der Erkrankung und der Multimorbidität,

2. angemessene Berücksichtigung der sozialen, lebensweltlichen und biographischen Bezüge,

3. aktivierende Behandlungsansätze, welche die Befähigung zur eigenständigen und selbstverantwortlichen Krankheitsbewältigung (Coping) fördern und damit Wege zur Verbesserung von Partizipation, Kompetenz und Information darstellen.

Dies zieht nach Meinung des Rates bei schwerwiegenden und komplexen chronischen Krankheiten, wie dem Asthma bronchiale und der COPD, ein in regelmäßigen Abständen durchgeführtes multidisziplinäres Assessment (Funktion, Aktivität, Partizipation) in angemessen ausgestatteten, kompetenten Einrichtungen nach sich.
Die Rehabilitation chronisch Kranker wird in der Erfüllung dieser Forderungen als eine selbstverständliche und ggf. wiederholte, den Lebenszyklen angepasste Kernaufgabe betrachtet. Die Durchführung der Rehabilitation habe sich dabei allein am Krankheitsverlauf und nicht an patientenfernen Verwaltungsrationalitäten zu orientieren. Für gesicherte Indikationen stehe die Rehabilitation auch nicht für Leistungsausschlüsse oder ausschlussgleiche Zusatzversicherungen zur Disposition.

Rehabilitation müsse je nach Phasenschweregrad der Krankheit und den jeweiligen Umständen und Präferenzen ambulant, teilstationär und stationär erfolgen. Intensivierte Gesundheitsförderung, Information, Schulung, Prävention und Rehabilitation des Patienten, sowie das routinemäßige Monitoring wichtiger Outcome- und Qualitätsindikatoren, ggf. auf der Basis evidenzbasierter Leitlinien werden dabei angemahnt.

Ebenso wie die Prävention zählt der Rat die Rehabilitation zu den vernachlässigten großen Unterversorgungsbereichen der chronisch Kranken. Dabei eröffne gerade die moderne Rehabilitation wie kaum ein anderer Behandlungsansatz die Chance auf eine umfassende und multidimensionale Versorgung chronisch Kranker. Rehabilitation sollte daher als essentieller Bestandteil der Versorgung Chronischkranker keine Ermessens- sondern eine Regelleistung sein.

Obwohl diese Defizite seit Jahren bekannt seien, fehlten in Deutschland nach wie vor ausreichende krankheitsphasenspezifische, zielgruppenorientierte und abgestufte Behandlungsangebote für unterschiedliche Patientengruppen, u.a. wohnortnahe ambulante oder teilstationäre Rehabilitationsstrukturen. Allerdings ist die Umsetzung solcher Forderungen durch die unzureichende Qualifikation auf Leistungserbringerseite gefährdet. Der Rat mahnt daher eine stärkere Praxisorientierung, Industrieunabhängigkeit und Verbindlichkeit der ärztlichen Fortbildung, die u.a. in der Aus- und Weiterbildung in Einrichtungen außerhalb der Akutversorgung ihren Ausdruck fände.

Nach Auffassung des Rates ist die Struktur der pneumologischen Versorgung zur Prävention, Kuration und Rehabilitation bei Asthma- und COPD-kranken Kindern und Erwachsenen in Deutschland verbesserungswürdig. Neben dem dringenden Anmahnen einer verbesserten pneumologischen Weiter- und Fortbildung, der Verbesserung der Strukturen für ambulante Patientenschulungs- und Rehabilitationsprogramme unter Berücksichtigung von Raucherentwöhnung, und Lungensportgruppen (Sport und Trainingstherapie), einschließlich der Regelung ihrer Finanzierung und Qualitätssicherung, der Schaffung einer größeren Zahl selbständiger, bettenführender Abteilungen an allgemeinen Krankenhäusern und von Lehrstühlen an jeder medizinischen Fakultät, plädiert der Rat für eine bessere Integration der pneumologischen Rehabilitation in alle Bereiche der pneumologischen Versorgung, sowie in die Lehre und Forschung. Dies sollte die Möglichkeit der Direkteinweisung in hochqualifizierte Rehabilitationskliniken, auch bei Exazerbationen chronischer, obstruktiver Lungenerkrankungen, und die Einführung von Case/ Disease-Management-Strategien für Problemgruppen von Patienten mit einschließen.

Es ist ein schwieriges Unterfangen, aufgrund einer rein auf Befragung aufgebauten Ermittlung allgemein gültige und quantitative 
Aussagen zur Über-, Unter- und Fehlversorgung im deutschen Gesundheitssystem zu machen. Der Rat selbst weist auf den eklatanten Mangel an belastbaren Gesundheitsdaten hin. Zudem liegen wissenschaftlich abgesicherte Angaben zu Wirksamkeit und Nutzen von Diagnostik und Behandlung nur für einen kleinen Teil medizinischer Prozeduren vor. Inzidenz und Prävalenz der gewählten Krankheitsgruppen beruhen eher auf Schätzungen, denn auf flächendeckend ermittelten „harten Zahlen“. Bevölkerungs- oder gruppenbezogene Daten zur Inanspruchnahme des Gesundheitswesens einschließlich der damit im Zusammenhang stehenden Kosten fehlen. Darüber hinaus mag der wissenschaftliche Purist grundsätzlich prospektiv gewonnene Primärdaten anmahnen. Dennoch gibt das Gutachten sehr eindrücklich die Situation wieder, in der sich Patienten mit Asthma und COPD und ihre Betreuer sowohl im Hinblick auf das somatische Leiden, als auch der Krankheitsfolgen (Funktion, Partizipation, generische und krankheitsbezogene Lebensqualität) in Deutschland befinden. Zur Klage wäre genügend Anlass, doch Selbsthilfe zur Abhilfe ist gefragt. Verbesserungen der ambulanten AsthmaSchulung und des Lungensportes sind zwischenzeitlich auf einen guten Weg gebracht $[2,5,7]$. Sie berücksichtigen nicht nur die Vorgaben der Evidence-based Medicine, sondern auch praktikable Strukturen der Qualitätssicherung.

Stationäre Maßnahmen sind unverändert das Standbein der pneumologischen Rehabilitation [6,7]. Sie sind wissenschaftlich begründet, qualitätsgesichert und finanziert. Allerdings sollten die, wenn auch kritisch zu betrachtenden Zahlen zu Inzidenz, Prävalenz und Morbidität eine höhere Einweisung in die stationäre Rehabilitation erwarten lassen. Die ambulante Rehabilitation wird zügig entwickelt [7]. Sie leidet allerdings an der Verfügbarkeit qualifizierten Personals, den harten, einer flächendeckenden Versorgung kurzfristig eher entgegenwirkenden, langfristig aber unabdingbar notwendigen Qualitätsnormen [9] und der mit Ausnahmen ungeklärten Frage der Finanzierung.

Ausbildung auf der ärztlichen Seite in Rehabilitationsfragen tut Not! Wer beherrscht denn die Fachsprache der Rehabilitaiton, die der International Classification of Impairments, Disabilities and Handicaps (ICIDH) entstammende Terminologie der Krankheitsfolgen, geschweige denn deren Assessment auf der Ebene der Primär- und der ambulanten Fachversorgung, d.h. am Ort der Indikations- und Zielfindung? Können die, die die Anwendung dieser Sprache erwarten, nämlich Kostenträger und medizinische Dienste, tatsächlich mit den Zuweisern verständlich kommunizieren? Wer denkt und handelt in den angemahnten vernetzten Strukturen der Prävention, Kuration und Rehabilitation? Sollten die vorgeschlagenen Verfahren des Case- und Disease-Management, ihr Potenzial sei unbestritten, aber zu wirksamen Maßnahmen der grundlegenden Verbesserung der Versorgung von Erwachsenen und Kindern mit Asthma und COPD in Deutschland werden, so müssen aus der Sicht der pneumologischen Rehabilitationsmedizin einige Visionen wirklich werden:

Wir benötigen die systematische, EBM-basierte, vorgeplante, zweckmäßig organisierte, qualitätsprüfbare, vernetzte Versorgung chronischer Lungen- und Atemwegserkrankungen (CaseManagement) $[3,4]$.

Die sektorale Organisation der Versorgung mit systemimmanenten Versorgungsbrüchen zu Lasten der chronisch Kranken muss einer durchlässigen Versorgungskette weichen, deren Schnittstellen bekannt sind und obligat bedient werden müssen. Dies muss für alle Kostenträgerarten Gültigkeit erlangen.

Zum Disease-Management befähigte Leitlinien auf der Grundlage der best available evidence sollten nicht Teilaspekte der Diagnostik und Therapie, sondern, wenn immer möglich, die Gesamtversorgung im Blick haben, da nur so obligate Versorgungskomponenten und -abläufe im Leben eines chronisch Lungen- und Atemwegskranken tatsächlich wirksam werden $[1,3,4]$.

Aktuell und konkret gilt es, die berechtigten Ansprüche des chronisch Kranken mit Asthma bronchiale und COPD in Leitlinien umzusetzen und auf diesem Wege auch dafür zu sorgen, dass Ärzte und Betreuer nicht nur in der angemessenen Zahl, sondern auch geforderten Qualifikation zur Verfügung stehen [1,9]. Im Hinblick auf die Umsetzung des Beschlussantrages für den 104. Deutschen Ärztetag 2001 in Ludwigshafen, TOP III: (Muster-) Weiterbildungsordnung für Ärztinnen und Ärzte, Novellierung der (Muster-) Weiterbildungsordnung, Richtlinien über die Inhalte, 13.C.7. Schwerpunkt Pneumologie, wünschen wir, dass die Ausbildung des zukünftigen Pneumologen die pneumologische Rehabilitation verstärkt berücksichtigt, beispielsweise durch ein genügend langes, in Einrichtungen der stationären pneumologischen Rehabilitation, d.h. v.a. außerhalb der Akutpneumologie stattfindendes Training. Dadurch könnten die nötigen Weichenstellungen für ein effizientes Management von Asthma und COPD einschließlich ihrer Krankheitsfolgen in integrierten, vernetzten Systemen in Praxis und Klinik Wirklichkeit werden [8].

\section{Literatur}

${ }^{1}$ Sachverständigenrat für die Konzertierte Aktion im Gesundheitswesen. Bedarfsgerechtigkeit und Wirtschaftlichkeit, Band III - Über-, Unter- und Fehlversorgung, Gutachten 200/2001. http://svr.de,

${ }^{2}$ Worth H, Meyer A, Folgering H, Kirsten D, Lecheler J, Magnussen H, Pleyer K, Schmidt S, Schmitz M, Taube K, Wettengl R. Empfehlungen der Deutschen Atemwegsliga zum Sport und körperlichen Training bei Patienten mit obstruktiven Atemwegserkrankungen. Pneumologie 2000; 54: 61-67

${ }^{3}$ KBV Kassenärztliche Bundesvereinigung. Disease-Management-Programm Asthma bronchiale (Entwurf) 24. Oktober 2001,

4 Petro W. BDA-Manual Asthma: Das Disease- und Case-Management des Asthma bronchiale. Pneumologische Rehabilitation, http:// www.ifap-index.de/bda-manuale/asthmacase/reha/index.html,

${ }^{5}$ Petro W (Hrsg). Pneumologische Prävention und Rehabilitation. Ziele - Methoden - Ergebnisse. Springer Verlag, Berlin, 2000

${ }^{6}$ Statement der Sektion Prävention und Rehabilitation (K. Bergmann, J. Fischer, M. Schmitz, F. Petermann, W. Petro). Die stationäre pneumologische Rehabilitation für Erwachsene. Zielsetzung - diagnostische und therapeutische Standards - Forschungsbedarf. Pneumologie, 1997; $51: 523-532$

${ }^{7}$ Statement der Sektion Prävention und Rehabilitation (R. Szczepanski, K.Taube, J, Junggeburth, H. Worth). Ambulante wohnortnahe pneumologische Prävention und Rehabilitation bei obstruktiven Atemwegserkrankungen - Stand, Perspektive und Forschungsbedarf. Pneumologie, 1998; 8, 52: $457-481$

8 Weiterbildung für Ärztinnen und Ärzte - Richtlinien über die Inhalte 13.C.7. Schwerpunkt Pneumologie Deutsche Gesellschaft für Pneumologie zusammen mit dem Bundesverband der Pneumologen. Entwurf vom 5. Dezember 2001 Berufs- und verbandspolitische Mitteilungen Bundesverband der Pneumologen. Dezember 2001. 54/01, 42 - 47

${ }^{9}$ Bundesarbeitsgemeinschaft für Rehabilitation. Rahmenempfehlung zur ambulanten kardiologischen Rehabilitation I. Allgemeiner Teil 9. Anforderungen an die ambulante Rehabilitationseinrichtung, 20. Oktober 2000, 21 - 30, http://www.bar-frankfurt.de 